Rodrigo Escalona ${ }^{1,2}$ and Jan Fawcett ${ }^{1}$

${ }^{1}$ Department of Psychiatry, University of New Mexico School of Medicine, Albuquerque, NM, USA; ${ }^{2} \mathrm{New}$ Mexico VA Health Care System, Albuquerque, NM, USA E-mail: jfawcett@salud.unm.edu

Contreras $F$, Prado C, Gonzalez $H$, Franz $D$ Osorio-Barrios F, Osorio F et al (2016). Dopamine receptor D3 signaling on CD4+ T cells favors Th1and Th17-mediated immunity. J Immunol 196: 4143-4149.

Fawcett J, Rush AJ, Vukelich J, Diaz SH, Dunklee L, Romo P et al (2016). Clinical experience with high dose pramipexole in treatment resistant mood disorder patients. Am J Psychiatry 173: 107-111.

Felger JC, Miller AH (2012). Cytokine effects on the basal ganglia and dopamine function: the subcortical source of inflammatory malaise. Front endocrinol 33: 315-327.

Lieberknecht V, Junqueira SC, Cunha MP, Barbosa TA, De Souza LF, Coelho IS et al (2016). Pramipexole, a dopamine D2/D3 receptor -preferring agonist prevents experimental autoimmune encephalomyelitis development in mice. $\mathrm{Mol}$ Neurobiol (e-pub ahead of print 22 January 2016). Swardfager W, Rosenblat JD, Benlamri M, McIntyre RS (2016). Mapping inflammation onto mood: inflammatory mediators of anhedonia. Neurosci Biobehav Rev 64: 148-166.

Uher R, Perlis RH, Henigsberg N, Zobel A, Rietschel M, Mors O et al (2012). Depression symptom dimensions as predictors of antidepressant treatment outcome: replicable evidence for interest-activity symptoms. Psychol Med 42 967-980.

Neuropsychopharmacology Reviews (2017) 42, 363-364; doi: 10.1 038/npp.2016.217

\section{Modeling the Impact of Social Contact on Substance Use}

Substance-use disorders remain resistant to most forms of clinical treatment. Although preclinical studies have identified many interventions that reliably decrease drug-seeking behavior in laboratory animals, only occasionally have they predicted successful outcomes in clinical trials. One common criticism of preclinical models is that most do not capture the complex social environment in which substance use occurs. The predictive validity of these models might be improved by incorporating relevant features of the social environment known to influence substance use.

Several investigators have recently introduced new models, or refined existing models, to capture aspects of the social environment that may influence drug-seeking behavior. Using the conditioned place preference procedure, a procedure in which a Pavlovian association is formed between a stimulus (eg, interoceptive drug cue, social partner) and a distinct environmental context, investigators have shown that drugs (eg, cocaine, nicotine) and social contact mutually enhance one another's rewarding effects when paired together (Thiel et al, 2009). Alternatively, social contact prevents and reverses a conditioned place preference for cocaine if the two are conditioned in separate contexts, and social contact reverses cocaine-induced increases in the transcription factor, Zif268, in the nucleus accumbens, amygdala, and ventral tegmental area (Fritz et al, 2011).

Advances in traditional drug selfadministration procedures are also allowing investigators to determine how the presence of a social partner influences drug intake. For instance, research with prairie voles, a socially monogamous species, has shown that alcohol intake decreases in the presence of a low-consumption social partner (Anacker et al, 2011). Our laboratory recently developed custombuilt, operant conditioning chambers that permit two rats to intravenously self-administer drugs simultaneously in the same chamber. We reported that the acquisition of cocaine selfadministration is facilitated in the presence of a partner that is also selfadministering cocaine (Smith et al, 2014) and the maintenance of cocaine self-administration is inhibited in the presence of a partner that does not have access to cocaine (Robinson et al, 2016). Interestingly, under conditions in which both partners have access to cocaine, individual patterns of cocaine intake become progressively more similar over time (Lacy et al, 2014). These studies suggest a number of behavioral mechanisms by which social contact may influence drug use. For instance, under conditions in which substance use is a group norm, group members may model drug use and selectively reinforce the drug use of others through social approval/ acceptance. In contrast, under conditions in which abstinence is a norm, drug use may be selectively punished through social rejection, and group members may model and differentially reinforce abstinence-related behaviors (see review by Strickland and Smith, 2014 for further examples and discussion).

The development of these animal models will significantly advance our understanding of the neurobiological mechanisms mediating the effects of social contact on substance use. Similar to the reinforcing effects of drugs, the reinforcing effects of social contact are dependent on dopamine transmission within the nucleus accumbens (Manduca et al, 2016), suggesting that this site may be a neuroanatomical locus in which information about social contact and drugs converge to mutually influence one another's rewarding effects. Consistent with this hypothesis, microinjection of the endogenous neuropeptide oxytocin into the nucleus accumbens increases social contact and affiliation ( $\mathrm{Yu}$ et al, 2016), and reduces drug self-administration and other measures of drug-seeking behavior (Baracz et al, 2016).

The discovery that information about drugs and the social environment converge on a common neurobiological locus furthers our understanding of preclinical, human laboratory, and epidemiological research showing that social contact can modify the reinforcing effects of drugs. This knowledge may also explain the past failure of preclinical studies (which artificially constrain the social environment) to consistently predict clinical trial outcomes (which generally do not control the social environment). By incorporating relevant features of social contact into our preclinical models, we should be able to better identify which interventions will successfully translate into clinical practice.

\section{FUNDING AND DISCLOSURE}

Work on this paper was funded by NIH Grant DA031725. The authors declare no conflict of interest. 
Mark A Smith ${ }^{1,2}$ and

Justin C Strickland ${ }^{3}$

${ }^{1}$ Department of Psychology, Davidson College, Davidson, NC, USA; ${ }^{2}$ Program in Neuroscience, Davidson College, Davidson, NC, USA; ${ }^{3}$ Department of Psychology, University of Kentucky, Lexington, KY, USA

E-mail: masmith@davidson.edu

Anacker AM, Loftis JM, Ryabinin AE (2011). Alcohol intake in prairie voles is influenced by the drinking level of a peer. Alcohol Clin Exp Res 35: 1884-1890.

Baracz SJ, Everett NA, McGregor IS, Cornish JL (2016). Oxytocin in the nucleus accumbens core reduces reinstatement of methamphetamineseeking behaviour in rats. Addict Biol 21: 316-325.

Fritz M, El Rawas R, Salti A, Klement S, Bardo MT, Kemmler $G$ et al (2011). Reversal of cocaineconditioned place preference and mesocorticolimbic Zif268 expression by social interaction in rats. Addict Biol 16: 273-284.

Lacy RT, Strickland JC, Smith MA (2014). Cocaine self-administration in social dyads using custombuilt operant conditioning chambers. J Neurosci Methods 236: 11-18.

Manduca A, Servadio M, Damsteegt R, Campolongo P, Vanderschuren LJ, Trezza V (2016). Dopaminergic neurotransmission in the nucleus accumbens modulates social play behavior in rats. Neuropsychopharmacology 41: 2215-2223.

Robinson AM, Lacy RT, Strickland JC, Magee CP, Smith MA (2016). The effects of social contact on cocaine intake under extended-access conditions in male rats. Exp Clin Psychopharmacol 24: 285-296.

Smith MA, Lacy RT, Strickland JC (2014). The effects of social learning on the acquisition of cocaine self-administration. Drug Alcohol Depend 141: 1-8.

Strickland JC, Smith MA (2014). The effects of social contact on drug use: behavioral mechansims controlling drug intake. Exp Clin Psychopharamcol 22: 23-34.

Thiel KJ, Sanabria F, Neisewander JL (2009). Synergistic interaction between nicotine and social rewards in adolescent male rats. Psychopharmacology 204: 391-402.

Yu CJ, Zhang SW, Tai FD (2016). Effects of nucleus accumbens oxytocin and its antagonist on social approach behavior. Behav Pharmacol (in press).

Neuropsychopharmacology Reviews (2017) 42, 364-365; doi: 10.1038/npp.2016.218

\section{Impact on Cortical Function of Cocaine Abuse Co-Occurring with HIV}

According to the USA Center for Disease Control (http://www.cdc.gov/) over 36 million humans are currently living with HIV infection, with 44000 new infections diagnosed annually in the USA, often reflecting risky sexual behavior. Substance use disorders (SUD) negatively influence every stage of the HIV/AIDS crisis, and SUD are a major obstacle in combating this global pandemic. Other than marijuana, cocaine is the most frequently abused illicit drug in HIV adults (eg, Martin et al., 2016). Cocaine use is associated with increased likelihood of engaging in risky sex, accelerated disease progression, and non-adherence to antiretroviral treatment. An emerging explanation for this association relates to impulsive decision-making. While both cocaine-abusing and HIVinfected individuals often score higher on cognitive impulsivity tasks, those with the comorbidity tend to show the greatest impulsivity (eg, Martin et al, 2016 and references therein). Human imaging studies point to impairments in prefrontal-subcortical networks in these deficits (eg, Meade et al, 2011). To capture a more mechanistic view and to help identify potential therapeutic targets, my laboratory recently set out to evaluate neuropathophysiology within the medial prefrontal cortex (mPFC) in the context of HIV/AIDS comorbidity. We focused on the principal outputs, ie, pyramidal neurons. As we recently reviewed (Wayman et al, 2015), our initial studies demonstrated that acute exposure to a prominent HIV-1 neurotoxic protein, Tat, induced an enduring $\mathrm{MPFC}$ astrogliosis and hyper excitability of mPFC pyramidal neurons that was exacerbated by chronic cocaine exposure. These effects were mediated by overactivation of high-voltage-activated L-type calcium channels and were associated with a Tat-induced increase expression of a calcium channel poreforming protein, Cav1.2- $\alpha 1 \mathrm{c}$. To better generalize the phenomenon to the chronic nature of brain exposure to HIV-1 proteins, we subsequently evaluated mPFC pathophysiology in HIV-1 transgenic rats following cocaine self-administration. As predicted by Tat-mediated excitotoxicity, the transgenic rats exhibited enhanced mPFC Cav1.2- $\alpha 1 \mathrm{c}$ expression, and a pyramidal cell hyper excitability and excitotoxicity (Wesley et al, 2016).
This dysregulation was exacerbated following 2 weeks of withdrawal from cocaine self-administration (Wesley et al., 2016).

These electrophysiological studies provide mechanistic support for a mPFC site of convergence for HIV-1 protein-mediated neurotoxicity and cocaine-induced dysregulation at the level of calcium channels within pyramidal neuronal outputs. Evidence for the behavioral consequences of such dysregulation is emerging, eg, in mice performing five-choice serial reactiontime tasks, DREADD-mediated stimulation of mPFC pyramidal neurons suppresses impulsivity (Warthen et al, 2016), and nifedipine (a brainpenetrant Cav1.2/Cav1.3 calcium channel antagonist) reduces impulsivity (Dudley et al, 2013). Thus, L-type calcium channel activity of mPFC pyramidal neurons may be an important regulator of impulsivity, with both 'too much' and 'too little' being detrimental. When viewed in the context of cognitive impulsivity in the HIV/AIDS cocaine-abuse co-morbid condition, these channels may be pharmacological targets for promoting healthy decision-making, and, in so doing, reduce HIV transmission and disease progression.

\section{FUNDING AND DISCLOSURE}

This research was supported by USPHSG DA033206 and DA033882, and the Center for Compulsive Behavior and Addiction at Rush University.

\section{T Celeste Napier ${ }^{1}$}

${ }^{1}$ Departments of Pharmacology and Psychiatry, Center for Compulsive Behavior and Addiction, Rush University Medical Center, Chicago, IL, USA

E-mail: Celeste_Napier@rush.edu

Dudley JA, Weir RK, Yan TC, Grabowska EM, Grimme AJ, Amini S et al (2013). Antagonism of L-type Cav channels with nifedipine differentially affects performance of wildtype and NK1R-/-mice in the 5-choice serial reaction-time task. Neuropharmacology 64: 329-336.

Martin E, Gonzalez R, Vassileva J, Maki PM, Bechara A, Brand M. (2016). Sex and HIV serostatus differences in decision making under risk among substance dependent individuals. J Clin Exp Neuropsychol 38: 404-415.

Meade CS, Lowen SB, MacLean RR, Key MD, Lukas SE. (2011). fMRI brain activation during a delay discounting task in HIV-positive adults with 